Article

\title{
On the Dirichlet Problem with Corner Singularity
}

\author{
Viktor A. Rukavishnikov *(D) and Elena I. Rukavishnikova \\ Computing Center of Far-Eastern Branch, Russian Academy of Sciences, Kim-Yu-Chen Str. 65, \\ 680000 Khabarovsk, Russia; rukavishnikova-55@mail.ru \\ * Correspondence: vark0102@mail.ru
}

Received: 23 September 2020; Accepted: 20 October 2020; Published: 29 October 2020

Abstract: We consider the Dirichlet problem for an elliptic equation with a singularity. The singularity of the solution to the problem is caused by the presence of a re-entrant corner at the boundary of the domain. We define an $R_{v}$-generalized solution for this problem. This allows for the construction of numerical methods for finding an approximate solution without loss of accuracy. In this paper, the existence and uniqueness of the $R_{v}$-generalized solution in set $\stackrel{\circ}{W}_{2, \alpha}^{1}(\Omega, \delta)$ is proven. The $R_{v}$-generalized solution is the same for different parameters $v$.

Keywords: corner singularity; boundary-value problem; $R_{\nu}$-generalized solution; existence and uniqueness solution

MSC: 35J25

\section{Introduction}

The weak solution of a boundary value problem for the elliptic equation in domain $\Omega$ $\left(\Omega \subset R^{2}\right)$ with reentrant corners $\gamma_{i}(i=1, \ldots, N)$ on boundary $\partial \Omega$, belongs to class $W_{2}^{1+k-\varepsilon}(\Omega)$, $k=\min _{i=1, \ldots, N}\left\{k_{i}\right\}$, where

$$
k_{i}= \begin{cases}\pi / \gamma_{i} & \text { for Dirichlet or Neuman problem, } \\ \pi / 2 \gamma_{i} & \text { for mixed boundary value problem, }\end{cases}
$$

and $\varepsilon$ is an arbitrary positive number.

For two-dimensional linear elliptic boundary-value problems with homogeneous Dirichlet-Dirichlet and Dirichlet-Neumann boundary conditions posed on the adjacent sides of re-entrant corners, it was stated that $0.25 \leq k_{i} \leq 0.67$ for $3 \pi / 2 \leq \gamma_{i} \leq 2 \pi$.

In this case, the approximate finite-element solution converges to a weak solution of the problem at an $O\left(h^{k}\right)$ rate ( $h$ is the mesh step) in the norm of Sobolev space $W_{2}^{1}(\Omega)$ [1,2].

By using meshes refined toward the singularity point or special methods for separating the singular and regular components of the solution near corner points, numerical methods of the first order of accuracy were created (see, for example, [3-12]). However, these methods lead to ill-conditioned systems of linear algebraic equations. These involve complication of computing process and affect the accuracy of the results.

For the boundary-value problem for elliptic equations with singularity, we propose to define its solutions as an $R_{v}$-generalized one (see, for examples, [13]). This conception allows for investigating problems with singularities of different kinds (discontinuous or not bounded coefficients, right hands of the equation and boundary conditions; existence of the re-entrant corners on the boundary) and constructing the weighted FEMfor these problems. This method provides convergence rates $O(h)$ and $O\left(h^{2}\right)$ of the approximate finite-element solution to the $R_{v}$-generalized one in the norm of the Sobolev and Lebesque weighted spaces, respectively (see [14-17]). 
For boundary-value problems with corner singularity, the existence and uniqueness of an $R_{v}$-generalized solution was established in [18-22] assuming that the coefficient multiplying $u(x)$ in the differential equation is sufficiently large. Below, this restrictive assumption is dropped and a weighted set of functions is defined in which a unique $R_{v}$-generalized solution exists for the given class of the boundary value problems; moreover, this solution is unique for all $v$.

\section{Problem Formulation}

Let $\Omega \subset R^{2}$ be a nonconvex polygon and $\partial \Omega$ its boundary, with an obtuse corner $\gamma$ with the vertex at the origin $O(0,0) ; \bar{\Omega}$ denotes the closure of $\Omega$, i.e. $\bar{\Omega}=\Omega \cup \partial \Omega$.

We introduce weight function

$$
\rho(x)= \begin{cases}\left(x_{1}^{2}+x_{2}^{2}\right)^{1 / 2}, & x \in \Omega^{\prime}, \\ \delta, & x \in \bar{\Omega} \backslash \Omega,\end{cases}
$$

where $\Omega^{\prime}=\left\{x \in \bar{\Omega}:\left(x_{1}^{2}+x_{2}^{2}\right)^{1 / 2} \leq \delta \ll 1\right\}$ (see Figure 1 ).

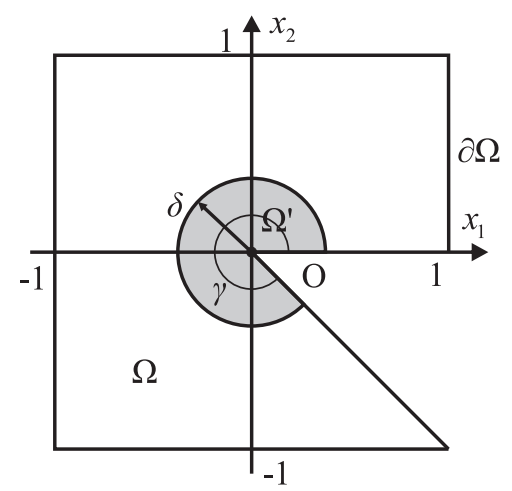

Figure 1. Domain $\Omega$ with re-entrant corner.

Let $W_{2, \alpha}^{1}(\Omega, \delta)(\alpha>0)$ be the set of functions subject the following conditions:

(a) $\left|D^{k} u(x)\right| \leq c_{1}(\delta / \rho(x))^{\alpha+k}$ for $x \in \Omega^{\prime}, k=0,1, c_{1}>0$;

(b) $\|u\|_{L_{2, \alpha}\left(\Omega \backslash \Omega^{\prime}\right)} \geq c_{2}>0$,

equipped with norm

$$
\|u\|_{W_{2, \alpha}^{1}(\Omega)}=\left(\sum_{|\lambda| \leq 1} \int_{\Omega} \rho^{2 \alpha}(x)\left|D^{\lambda} u(x)\right|^{2} d x\right)^{1 / 2},
$$

where $D^{\lambda}=\frac{\partial^{|\lambda|}}{\partial x_{1}^{\lambda_{1}} \partial x_{2}^{\lambda_{2}}}, \lambda=\left(\lambda_{1}, \lambda_{2}\right)$ with $|\lambda|=\lambda_{1}+\lambda_{2}$ (here, $\lambda_{1}$ and $\lambda_{2}$ are non-negative integers). For $k=0$, we use notation $W_{2, \alpha}^{0}(\Omega, \delta)=L_{2, \alpha}(\Omega, \delta)$.

Set $\stackrel{\circ}{W}_{2, \alpha}^{1}(\Omega, \delta) \subset W_{2, \alpha}^{1}(\Omega, \delta)$ is a closure in Norm (1) of set $C_{\infty}(\Omega, \delta)$ that contain functions satisfying Conditions (a) and (b). Here, $C_{\infty}(\Omega)$ is an infinitely differentiable and finite space in $\Omega$ functions.

Let $L_{\infty,-\alpha}\left(\Omega, c_{3}\right)$ denote the set of functions with norm

$$
\|u\|_{L_{\infty,-\alpha}\left(\Omega, c_{3}\right)}=\underset{x \in \Omega}{\operatorname{vraimax}}\left|\rho^{-\alpha}(x) u(x)\right| \leq c_{3},
$$

where $c_{3}$ is positive constant not depending on $u(x)$.

We consider differential equation 


$$
-\sum_{l=1}^{2} \frac{\partial}{\partial x_{l}}\left(a_{l l}(x) \frac{\partial u}{\partial x_{l}}\right)+a(x) u(x)=f(x), x \in \Omega,
$$

with boundary condition

$$
u(x)=0, \quad x \in \partial \Omega .
$$

Definition 1. Boundary-value problem (2), (3) is called the Dirichlet problem with uncoordinated degeneration of the input data if the coefficients and right-hand side of the equation satisfy conditions

$$
\begin{gathered}
a_{l l} \in L_{\infty,-\beta}\left(\Omega, c_{4}\right), \quad a \in L_{\infty,-\beta}\left(\Omega, c_{5}\right), \\
\sum_{l=1}^{2} a_{l l}(x) \xi_{l}^{2} \geq c_{6} \rho^{\beta}(x) \sum_{l=1}^{2} \xi_{l}^{2}, \\
a(x)>c_{7} \rho^{\beta}(x) \text { almost everywhere on } \Omega, \\
f \in L_{2, \mu}(\Omega, \delta), \quad \mu \geq 0 .
\end{gathered}
$$

Here $\beta, \mu$ are real numbers, $c_{i} \geq 0, i=4,5,6,7$, while $\xi_{1}$ and $\xi_{2}$ are arbitrary real parameters.

Define

$$
\begin{gathered}
a_{\Omega}(u, v)=\int_{\Omega}\left[\sum_{l=1}^{2} a_{l l} \rho^{2 v} \frac{\partial u}{\partial x_{l}} \frac{\partial v}{\partial x_{l}}+a_{l l} \frac{\partial \rho^{2 v}}{\partial x_{l}} \frac{\partial u}{\partial x_{l}} v\right]+a \rho^{2 v} u v d x \\
f_{\Omega}(v)=\int_{\Omega} \rho^{2 v} f v d x
\end{gathered}
$$

Definition 2. Function $u_{v}$ from space $\stackrel{\circ}{W}_{2, v+\beta / 2}^{1}(\Omega)$ is called an $R_{v}$-generalized solution of the Dirichlet problem with uncoordinated degeneration of the input data if identity

$$
a_{\Omega}\left(u_{v}, v\right)=f_{\Omega}(v)
$$

holds for all $v \in \stackrel{\circ}{W}_{2, v+\beta / 2}^{1}(\Omega)$ and for any given value of $v$, satisfying inequality

$$
v \geq \mu+\beta / 2
$$

Remark 1. The summands of bilinear form $a_{\Omega}(u, v)$ have different asymptotic orders. This is due to the fulfilment of Condition (4). In $[18,19]$ for such problems, we established that there is no unique $R_{v}$-generalized solution in space $\stackrel{\circ}{W}_{2, \alpha}^{1}(\Omega)$, since there is a sheaf of solutions in the neighborhood of the singular point. In [20-22], we proved the uniqueness of the $R_{v}$-generalized solution in set $\stackrel{\circ}{W}_{2, \alpha}^{1}(\Omega, \delta)$ with the correct choice of parameters $\checkmark$ and $\delta$.

Examples of problems with uncoordinated degeneration of the initial data can serve as boundary-value problems for the Lamé and Maxwell systems, and Stokes problem in domains with re-entrant corners on the boundary.

Remark 2. The main idea of the proposed approach is as follows: we introduce into the generalized statement of Problems (2)-(7) a special weight function $\rho$ raised to a certain power depending on the spaces that contain the coefficients and the right-hand side of the equation, sizes of re-entrant corners on the domain boundary. 
The presence of such a function in the definition of the solution overpowers the singularity and ensures that the $R_{v}$-generalized solution of the problem belongs to set $W_{2, v}^{2}(\Omega, \delta)$.

\section{Existence and Uniqueness of $R_{v}$-Generalized Solution}

We formulate the main result.

Theorem 1. Let Conditions (4)-(8) hold and

$$
v+\frac{\beta}{2}>0 .
$$

Then, for any $v$ satisfying Conditions (8) and (9), there always exists parameter $\delta$, such that $R_{v}$-generalized solution $u_{v}$ of the Dirichlet problem with uncoordinated degeneration of the input data exists and is unique from set $\stackrel{\circ}{W}_{2, v+\beta / 2}^{1}(\Omega, \delta)$ and

$$
\left\|u_{v}\right\|_{W_{2, v+\beta / 2}^{1}(\Omega, \delta)} \leq c_{8}\|f\|_{L_{2, u}(\Omega, \delta)},
$$

where a positive constant $c_{8}$ is independent of $f$ and $u_{v}$.

To prove this theorem, we need the lemma.

Lemma 1 ([22]). For each function $u$ in set $W_{2, \alpha}^{1}(\Omega, \delta)$ and for any $\alpha^{*}>\alpha$, estimate

$$
\|u\|_{L_{2, \alpha^{*}-1}\left(\Omega^{\prime}, \delta\right)} \leq c_{9}\|u\|_{L_{2, \alpha^{*}}(\Omega, \delta)}
$$

holds, where $c_{9}=c_{10} \frac{\delta^{\alpha}}{\sqrt{\alpha^{*}-\alpha}}, c_{10}=$ const $>0$.

Proof. For $\alpha^{*}>\alpha$ and Condition (a), we have

$$
\|u\|_{L_{2, \alpha^{*}-1}\left(\Omega^{\prime}, \delta\right)}^{2}=\int_{\Omega^{\prime}} \rho^{2\left(\alpha^{*}-1\right)} u^{2} d x \leq c_{1}^{2} \delta^{2 \alpha} \int_{\Omega^{\prime}} \rho^{2\left(\alpha^{*}-1\right)} \rho^{-2 \alpha} d x \leq \frac{c_{1}^{2} \delta^{2 \alpha} c_{11} \delta^{2\left(\alpha^{*}-\alpha\right)}}{2\left(\alpha^{*}-\alpha\right)},
$$

where $c_{11}$ is a constant dependent of mes $\Omega^{\prime}$. From Condition (b) for function $u$, we obtain

$$
\|u\|_{L_{2, \alpha^{*}}(\Omega)}^{2} \geq\|u\|_{L_{2, \alpha^{*}}\left(\Omega \backslash \Omega^{\prime}\right)}^{2}=\delta^{2\left(\alpha^{*}-\alpha\right)}\|u\|_{L_{2, \alpha}\left(\Omega \backslash \Omega^{\prime}\right)}^{2} \geq c_{2}^{2} \delta^{2\left(\alpha^{*}-\alpha\right)} .
$$

From Inequalities (12) and (13), we obtain estimate (11) with $c_{10}=\frac{c_{1}}{c_{2}} \sqrt{\frac{c_{11}}{2}}$.

Proof of Theorem 1. First, we show that forms $a_{\Omega}(u, v)$ and $f_{\Omega}(v)$ are continuous on $\stackrel{\circ}{W}_{2, v+\beta / 2}^{1}(\Omega, \delta)$, and inequalities

$$
\begin{gathered}
a_{\Omega}(u, v) \leq c_{12}\|u\|_{W_{2, v+\beta / 2}^{1}(\Omega, \delta)}\|v\|_{W_{2, v+\beta / 2}^{1}(\Omega, \delta)^{\prime}} \\
f_{\Omega}(v) \leq c_{13}\|f\|_{L_{2, u}(\Omega, \delta)}\|v\|_{W_{2, v+\beta / 2}^{1}(\Omega, \delta)}
\end{gathered}
$$

hold. The proofs of Estimates (14) and (15) are established by using Conditions (4), (7), and (8), Lemma 1, and Cauchy-Schwarz inequality.

Let us show that the bilinear form is $\stackrel{\circ}{W}_{2, v+\beta / 2}^{1}$-elliptical in $\Omega$. We have

$$
a_{\Omega}(u, u)=\sum_{k=1}^{2} \int_{\Omega}\left[a_{k k} \rho^{2 v}\left(\frac{\partial u}{\partial x_{k}}\right)^{2}+a_{k k} \frac{\partial \rho^{2 v}}{\partial x_{k}} \frac{\partial u}{\partial x_{k}} u\right] d x+\int_{\Omega} a \rho^{2 v} u^{2} d x
$$


for any $u$ from $\stackrel{\circ}{W}_{2, v+\beta / 2}^{1}(\Omega, \delta)$. Considering Condition (4), we establish the inequality for the absolute value of the second term on the right-hand side in (16)

$$
\begin{aligned}
\left|\sum_{k=1}^{2} \int_{\Omega} a_{k k} \frac{\partial \rho^{2 v}}{\partial x_{k}} \frac{\partial u}{\partial x_{k}} u d x\right| \leq\left|\sum_{k=1}^{2} \int_{\Omega^{\prime}} a_{k k} 2 v \rho^{2 v-1} \frac{\partial u}{\partial x_{k}} u d x\right| \leq \\
\leq \varepsilon \sum_{k=1}^{2} \int_{\Omega^{\prime}} \rho^{2 v+\beta}\left(\frac{\partial u}{\partial x_{k}}\right)^{2} d x+\frac{2 c_{4}^{2} v^{2}}{\varepsilon} \int_{\Omega^{\prime}} \rho^{2 v+\beta-1} u^{2} d x
\end{aligned}
$$

From (16) and the last inequality, we obtain

$$
\begin{aligned}
a_{\Omega}(u, u) \geq \sum_{k=1}^{2} \int_{\Omega} a_{k k} \rho^{2 v}\left(\frac{\partial u}{\partial x_{k}}\right)^{2} d x-\varepsilon \sum_{k=1}^{2} \int_{\Omega^{\prime}} \rho^{2 v+\beta}\left(\frac{\partial u}{\partial x_{k}}\right)^{2} d x+ & \\
& +\int_{\Omega} a \rho^{2 v} u^{2} d x-\frac{2 c_{4}^{2} v^{2}}{\varepsilon} \int_{\Omega^{\prime}} \rho^{2 v+\beta-1} u^{2} d x
\end{aligned}
$$

Supposing that $\alpha^{*}$ and $\alpha$ equal $v$ and $v / 2$ in Lemma 1 , respectively, we have

$$
\|u\|_{L_{2, v+\beta / 2-1}\left(\Omega^{\prime}, \delta\right)}^{2} \leq \frac{2 c_{10}^{2} \delta^{v}}{v}\|u\|_{L_{2, v+\beta / 2}(\Omega, \delta)^{\prime}}^{2} \quad c_{10}=\text { const }>0 .
$$

Considering (5), (6) and (18) from Estimate (17) we obtain

$$
a_{\Omega}(u, u) \geq\left(c_{5}-\varepsilon\right)|u|_{W_{2, v+\beta / 2}^{1}(\Omega, \delta)}^{2}+\left(c_{6}-\frac{4 c_{4}^{2} c_{10}^{2} v \delta^{v}}{\varepsilon}\right)\|u\|_{L_{2, v+\beta / 2}(\Omega, \delta)}^{2} .
$$

Obviously, we can always choose $\varepsilon$ and $\delta$, such that constants $c_{5}>\varepsilon, c_{6}>\frac{4 c_{4}^{2} c_{10}^{2} \nu \delta^{v}}{\varepsilon}$, and inequality

$$
a_{\Omega}(u, u) \geq c_{14}\|u\|_{W_{2, v+\beta / 2}^{1}}^{2}(\Omega, \delta)
$$

is valid with constant $c_{14}=\min \left(c_{6}-\varepsilon, c_{7}-\frac{4 c_{4}^{2} c_{10}^{2} v \delta^{v}}{\varepsilon}\right)$. Therefore, bilinear form $a_{\Omega}(u, u)$ is $\stackrel{\circ}{W}{ }_{2, v+\beta / 2}^{1}$-elliptical.

According to (14), (15), and (19), bilinear form $a_{\Omega}(u, u)$ is continuous, and $\stackrel{\circ}{W}{ }_{2, v+\beta / 2}^{1}$-elliptical, and linear form $f_{\Omega}(v)$ is continuous on $\stackrel{\circ}{W}_{2, v+\beta / 2}^{1}(\Omega, \delta)$; then, the existence and uniqueness of an $R_{v}$-generalized solution of Problems (2)-(7) follows from the Lax-Milgram theorem (see [1]).

Considering that

$$
c_{14}\left\|u_{v}\right\|_{W_{2, v+\beta / 2}^{1}(\Omega, \delta)}^{2} \leq a_{\Omega}\left(u_{v}, u_{v}\right)=f_{\Omega}\left(u_{v}\right) \leq c_{13}\left\|u_{v}\right\|_{W_{2, v+\beta / 2}^{1}(\Omega, \delta)}\|f\|_{L_{2, \mu}(\Omega, \delta)}
$$

we obtain Estimate (10).

Theorem 2 ([23]). If for some $\delta$ there is a set of values $v$, such that an $R_{v}$-generalized solution of Problems (2)-(7) exists in set $\stackrel{\circ}{W}_{2, v+\beta / 2}^{1}(\Omega, \delta)$, then this solution is unique for all such $v$.

Remark 3. Theorems 1 and 2 are valid for the domain with a boundary containing multiple re-entrant corners.

Remark 4. For the Stokes problem, the Lamé system, and Maxwell's equations in a two-dimensional domain with a boundary containing re-entrant corners, we constructed and investigated a weighted FEM that provides convergence rate $\mathrm{O}(h)$, and this rate does not depend of the singularity size (value) $([14-17,23,24])$. The solution for those boundary-value problems was defined as an $R_{v}$-generalized one in the weighted set. 


\section{Conclusions}

We presented the concept of an $R_{v}$-generalized solution for boundary-value problems with a singularity. We proved a theorem on the existence and uniqueness of an $R_{v}$-generalized solution in a special set. This definition of the solution allows for creating highly efficient numerical methods without loss of accuracy for boundary-value problems with a singularity. The established theorem helps to determine optimal parameters $v$ and $\delta$ in numerical methods to improve the accuracy of finding an approximate solution. We plan to determine the body of optimal parameters (BOP) for the crack problem, for boundary-value problems of the theory of elasticity in regions with re-entrant angles at the boundary, and with a change in the type of boundary conditions.

Author Contributions: V.A.R. and E.I.R. contributed equally in each stage of the work. All authors read and approved the final version of the paper. All authors have read and agreed to the published version of the manuscript.

Funding: This research received no external funding.

Acknowledgments: We would like to thank the Academician of the Russian Academy of Sciences V.A. Il'in for the invaluable suggestions due to which the manuscript was significantly improved.

Conflicts of Interest: The authors declare no conflict of interest.

\section{References}

1. Ciarlet, P.G. The Finite Element Method for Elliptic Problems; Studies in Mathematics and its Applications; North-Holland: Amsterdam, The Netherlands, 1978; 530p.

2. Samarskii, A.A.; Lazarov, R.D.; Makarov, V.L. Finite Difference Schemes for Differential Equations with Generalized Solutions; Visshaya Shkola: Moscow, Russia, 1987; 296p.

3. Bramble, J.H.; Habburd, B.E. Approximation of solutions of mixed boundary value problems for Poisson's equation by finite differences. J. Assoc. Comput. Math. 1965, 1, 114-123. [CrossRef]

4. Fix, G.J. Higher-order Rayleigh-Ritz approximations. J. Math. Mech. 1969, 18, 645-657.

5. Grisvard, P. Elliptic Problems in Nonsmooth Domains; Pitman: Boston, MA, USA, 1985; 410p.

6. Brenner, C. Multigrid methods for the computation of singular solutions and stress intensity factors I: Corner singularities. Math. Comput. 1999, 68, 559-583. [CrossRef]

7. Lee, E.; Manteuffel, T.A.; Westphal, C.R. Weighted-norm first-order system least squares (FOSLS) for problems with corner singularities. SIAM J. Numer. Anal. 2006, 44, 1974-1996. [CrossRef]

8. Li, H.; Mazzucato, A.; Nistor, V. Analysis of the finite element method for transmission/mixed boundary value problems on general polygonal domains. Electron. Trans. Numer. Anal. 2010, 37, 41-69.

9. Egger, H.; Rüde, U.; Wohlmuth, B. Energy-corrected finite element methods for corner singularities. SIAM J. Numer. Anal. 2014, 52, 171-193. [CrossRef]

10. Jhon, L.; Pustejovska, P.; Wohlmuth, B.; Rüde, U. Energy-corrected finite element methods for the Stokes system. IMA J. Numer. Anal. 2017, 37, 687-729. [CrossRef]

11. Francis, A.; Ortiz-Bernardin, A.; Bordas, S.P.; Natarajan, S. Linear smoothed polygonal and polyhedral finite elements. Int. J. Numer. Meth. Eng. 2017, 109, 1263-1288. [CrossRef]

12. Ma, W.; Liu, G.; Ma, H. A smoothed enriched meshfree Galerkin method with two-level nesting triangular sub-domains for stress intensity factors at crack tips. Theor. Appl. Fract. Mech. 2019, 101, 279-293. [CrossRef]

13. Rukavishnikov, V.A. Methods of numerical analysis for boundary value problem with strong singularity. Russ. J. Numer. Anal. Math. Model. 2009, 24, 565-590. [CrossRef]

14. Rukavishnikov, V.A.; Rukavishnikova, E.I. The finite element method for the first boundary value problem with compatible degeneracy of the input data. Dokl. Akad. Nauk 1994, 338, 731-733.

15. Rukavishnikov, V.A.; Rukavishnikova, H.I. The finite element method for a boundary value problem with strong singularity. J. Comput. Appl. Math. 2010, 234, 2870-2882. [CrossRef]

16. Rukavishnikov, V.A.; Rukavishnikova, H.I. On the error estimation of the finite element method for the boundary value problems with singularity in the Lebesgue weighted space. Numer. Funct. Anal. Optim. 2013, 34, 1328-1347. [CrossRef] 
17. Rukavishnikov, V.A.; Rukavishnikov, A.V. Weighted finite element method for the Stokes problem with corner singularity. J. Comput. Appl. Math. 2018, 341, 144-156. [CrossRef]

18. Rukavishnikov, V.A. The Dirichlet problem with incompatible degeneration of initial data. Dokl. Akad. Nauk 1994, 337, 447-449.

19. Rukavishnikov, V.A. The Dirichlet problem for a second-order elliptic equation with noncoordinated degeneration of the input data. Differ. Equ. 1996, 32, 406-412.

20. Rukavishnikov, V.A. On the uniqueness of the $R_{v}$-generalized solution of boundary value problems with noncoordinated degeneration of the initial data. Dokl. Math. 2001, 63, 68-70.

21. Rukavishnikov, V.A. On the existence and uniqueness of an $R_{v}$-generalized solution of a boundary value problem with uncoordinated degeneration of the input data. Dokl. Math. 2014, 90, 562-564. [CrossRef]

22. Rukavishnikov, V.A.; Rukavishnikova, E.I. Existence and uniqueness of an $R_{v}$-generalized solution of the Dirichlet problem for the Lamé system with a corner singularity. Differ. Equ. 2019, 55, 832-840. [CrossRef]

23. Rukavishnikov, V.A.; Kuznetsova, E.V. Coercive estimate for a boundary value problem with noncoordinated degeneration of the data. Differ. Equ. 2007, 43, 550-560. [CrossRef]

24. Rukavishnikov, V.A.; Mosolapov, A.O. New numerical method for solving time-harmonic Maxwell equations with strong singularity. J. Comput. Phys. 2012, 231, 2438-2448. [CrossRef]

Publisher's Note: MDPI stays neutral with regard to jurisdictional claims in published maps and institutional affiliations.

(C) 2020 by the authors. Licensee MDPI, Basel, Switzerland. This article is an open access article distributed under the terms and conditions of the Creative Commons Attribution (CC BY) license (http:/ / creativecommons.org/licenses/by/4.0/). 